\title{
No evidence of a hot Jupiter around HD $188753 A^{\star, \star \star}$
}

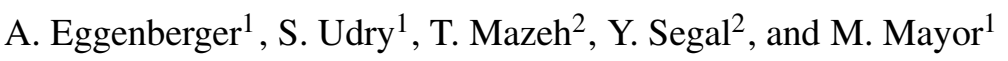 \\ 1 Observatoire de Genève, 51 Ch. des Maillettes, 1290 Sauverny, Switzerland \\ e-mail: Anne.Eggenberger@obs.unige.ch \\ 2 School of Physics and Astronomy, Raymond and Beverly Sackler Faculty of Exact Sciences, Tel Aviv University, Tel Aviv, Israel \\ Received 28 November 2006 / Accepted 25 January 2007
}

\section{ABSTRACT}

\begin{abstract}
Context. The discovery of a short-period giant planet (a hot Jupiter) around the primary component of the triple star system HD 188753 has often been considered as an important observational evidence and as a serious challenge to planet-formation theories.

Aims. Following this discovery, we monitored HD 188753 during one year to better characterize the planetary orbit and the feasibility of planet searches in close binaries and multiple star systems.

Methods. We obtained Doppler measurements of HD 188753 with the ELODIE spectrograph at the Observatoire de Haute-Provence. We then extracted radial velocities for the two brightest components of the system using our multi-order, two-dimensional correlation algorithm, TODCOR.

Results. Our observations and analysis do not confirm the existence of the short-period giant planet previously reported around HD 188753 A. Monte Carlo simulations show that we had both the precision and the temporal sampling required to detect a planetary signal like the one quoted.

Conclusions. From our failure to detect the presumed planet around HD 188753 A and from the available data on HD 188753, we conclude that there is currently no convincing evidence of a close-in giant planet around HD $188753 \mathrm{~A}$.
\end{abstract}

Key words. techniques: radial velocities - stars: binaries: spectroscopic - stars: individual: HD 188753 - stars: planetary systems

\section{Introduction}

In a recent paper, Konacki (2005a) has reported the discovery of a 3.35-day radial-velocity modulation of HD $188753 \mathrm{~A}$ with a semiamplitude of $149 \mathrm{~m} \mathrm{~s}^{-1}$, and attributed this signal to the presence of a planet with a minimum mass of $1.14 M_{\text {Jup }}$ in orbit around the star. Interestingly, HD $188753 \mathrm{~A}$ is the primary component of a triple star system, while the visual companion, HD $188753 \mathrm{~B}$, is itself a spectroscopic binary with a period of 155 days (Griffin 1977; Konacki 2005a). The visual orbit of the AB pair is characterized by a period of 25.7 years, a semimajor axis of $12.3 \mathrm{AU}$ (0.27" separation), and an eccentricity of 0.5 (Söderhjelm 1999). HD 188753 is therefore a hierarchical system, with the primary component (A) hosting the giant planet and the secondary $(\mathrm{Ba})$ and tertiary $(\mathrm{Bb})$ components forming a close pair in orbit at a distance of 12.3 AU from the planet-host star.

The discovery of a giant planet around HD $188753 \mathrm{~A}$ has attracted much attention, as the proximity of HD $188753 \mathrm{~B}$ poses a serious problem for planet formation theories. Indeed, the periastron distance of the visual binary is only $6.2 \mathrm{AU}$, so that any protoplanetary disk around HD 188753 A would be truncated at $\sim 2$ AU (Konacki 2005a; Jang-Condell 2007), i.e. probably inside the snow line. The favored core accretion model, which stipulates that the cores of giant planets form beyond the snow line, might thus have a problem accounting for the existence of a giant planet around HD 188753 A. Assuming that the system has

* Based on observations collected at the Observatoire de HauteProvence with the ELODIE echelle spectrograph mounted on the 1.93-m telescope.

$\star \star$ Table 1 is only available in electronic form at http://www. aanda.org maintained its current orbital configuration ever since the planet formed, explaining the existence of a hot Jupiter around the primary component of HD 188753 remains a challenge (Nelson 2000; Mayer et al. 2005; Boss 2006; Jang-Condell 2007) and might indicate that the core accretion mechanism is not able to account for the existence of all the planets discovered so far.

An alternative way to explain the existence of a close-in Jovian planet around HD $188753 \mathrm{~A}$ and also to circumvent the theoretical problem is to assume that the current orbital configuration is the result of a dynamical process (Pfahl 2005; Portegies Zwart \& McMillan 2005). According to this approach, HD 188753 A was a single star or had a more distant stellar companion at the planet formation phase, and this initial state was later transformed through a dynamical encounter into the triple system we presently observe. Pfahl \& Muterspaugh (2006) estimate that dynamical interactions could deposit giant planets in about $0.1 \%$ of the binaries with a semimajor axis $a<50 \mathrm{AU}$. The existence of a short-period Jovian planet around HD 188753 A might thus be the result of a dynamical process, rather than of an in situ formation (Pfahl 2005; Portegies Zwart \& McMillan 2005; Jang-Condell 2007).

Among the 200 extrasolar planets discovered so far by Doppler spectroscopy, 33 are known to orbit the components of binaries or triple stars (Eggenberger et al. 2004; Mugrauer et al. 2005; Raghavan et al. 2006). However, unlike the giant planet around HD $188753 \mathrm{~A}$, most of these planets reside in systems with separations larger than 100 AU. Since double stars closer than 2-6" present observational difficulties for Doppler studies, they have commonly been left out of radial-velocity planet searches. The occurrence of planets in binaries with semimajor axes below $100 \mathrm{AU}$ is therefore still largely unprobed. Despite this strong bias and prior to the discovery by Konacki, 
three planets were found in stellar systems with separations close to 20 AU: Gliese 86 (Queloz et al. 2000; Els et al. 2001; Mugrauer \& Neuhäuser 2005; Lagrange et al. 2006), $\gamma$ Cephei (Hatzes et al. 2003; Neuhäuser et al. 2007; Torres 2007), and HD 41004 (Zucker et al. 2003, 2004). Two features render the planet around HD 188753 A particularly interesting. First, both the planetary and the binary orbital parameters are known (only $\gamma$ Cephei shares this property). Second, the binary periastron distance may be small enough to preclude giant planet formation according to the canonical models.

Doppler searches for planets in close binaries have recently proven feasible using dedicated reduction techniques based on two-dimensional correlation (Zucker et al. 2003; Konacki 2005 b). In order to probe the occurrence of planets in close stellar systems, two surveys searching for planets in spectroscopic binaries are currently underway (Eggenberger et al. 2003; Konacki 2005b), the planet around HD 188753 A being a product of Konacki's survey. Yet, deriving the velocity of HD $188753 \mathrm{~A}$ to the precision needed to reveal the presence of a planet is particularly challenging, as the radial velocity of the secondary, HD $188753 \mathrm{Ba}$, varies with a semiamplitude of $\sim 13 \mathrm{~km} \mathrm{~s}^{-1}$ over a timescale of 155 days.

In order to study this intriguing system further, we monitored HD 188753 during one year with the ELODIE spectrograph (Baranne et al. 1996). We then used our multi-order TODCOR algorithm (Zucker et al. 2003) to derive the radial velocities of the two brightest components, namely HD $188753 \mathrm{~A}$ and HD $188753 \mathrm{Ba}$. Unfortunately, our data and analysis do not confirm the existence of the planet reported by Konacki (2005a) around HD $188753 \mathrm{~A}$. We present our observations and data reduction technique in Sect. 2. Our results and the lack of evidence of a hot Jupiter around HD 188753 A are described in Sect. 3 and discussed in Sect. 4.

\section{Observations and data analysis}

We observed HD 188753 with the ELODIE echelle spectrograph (Baranne et al. 1996) at the Observatoire de Haute-Provence (France) between July 2005 and August 2006. Altogether, we gathered 48 spectra with a typical signal-to-noise ratio of 55 (per pixel at $550 \mathrm{~nm}$ ). Note that ELODIE is a fiber-fed spectrograph with a fiber of $2^{\prime \prime}$ in diameter. As the angular separation between HD 188753 A and HD 188753 B is always less than $0.4^{\prime \prime}$, our observations necessarily record the combined spectrum of the whole system. In the course of the observations, we realized that the precision seemed degraded when the two brightest stars (A and $\mathrm{Ba}$ ) had similar velocities (velocity difference of less than a few $\mathrm{km} \mathrm{s}^{-1}$ ), and we subsequently avoided observing the system at those particular phases of the 155-day modulation.

Our spectra were reduced and cross-correlated online, the wavelength calibration being provided by the thorium-argon simultaneous reference technique (Baranne et al. 1996). The double-lined nature of HD 188753 was evident at the telescope, where two blended correlation features could be seen, one corresponding to component $\mathrm{A}$, the other corresponding to component $\mathrm{Ba}$. The contribution of the third component $(\mathrm{Bb})$ to the total flux is quite modest, so that the system can basically be considered as a double-lined spectroscopic binary. Nonetheless, extracting precise radial velocities for the two brightest stars of the system is challenging, for two main reasons: (i) the difference in mean velocity between components $\mathrm{A}$ and $\mathrm{Ba}$ is currently only of $2 \mathrm{~km} \mathrm{~s}^{-1}$; (ii) the radial velocity of component $\mathrm{Ba}$ varies with a period of 155 days and a semiamplitude of $\sim 13 \mathrm{~km} \mathrm{~s}^{-1}$, which is not very different from the sum of the intrinsic widths of the two correlation features. This leads to a situation where the two correlation features are always strongly blended, the blend changing continuously due to the 155-day modulation.

Given the double-lined nature of HD 188753 and the strong line blending, we derived radial velocities using a multi-order version (Zucker et al. 2003) of the two-dimensional correlation algorithm TODCOR (Zucker \& Mazeh 1994). This algorithm uses two templates with a given flux ratio and unknown Doppler shifts to compute the two-dimensional correlation function, whose maximum simultaneously gives the radial velocity of both components. Our version of TODCOR uses as templates high signal-to-noise stellar spectra built up using spectra from our planet search programs with CORALIE and ELODIE (Queloz et al. 2000; Perrier et al. 2003), along with spectra from the surveys for low-mass companions to $\mathrm{M}$ dwarfs by Delfosse and coworkers (Delfosse et al. 1998, 1999). Each template can furthermore be convolved with a rotational broadening profile, thus allowing for a better fit to the observed spectrum. In the multi-order version of TODCOR, the flux ratio is a function of wavelength and is calculated for each order according to the spectral types of the two templates using the library of spectral energy distributions by Pickles (1998). To insure fine-tuning with the observed system, the table of flux ratios can also be multiplied by a global normalization factor.

To derive the radial velocities of HD $188753 \mathrm{~A}$ and $\mathrm{Ba}$, we ran TODCOR for a variety of different pairs of templates from our library, searching for the pair that best matches our observed composite spectra. The two templates finally retained were the spectrum of HD 224752 (a G6 dwarf) for HD 188753 A and the spectrum of HD 225208 (a K0 dwarf) for HD $188753 \mathrm{Ba}$. This template configuration was chosen because (i) it gave the lowest residuals for the spectroscopic orbit of component $\mathrm{Ba}$, and (ii) the primary template maximized the correlation coefficient for component A. Additional fine-tuning consisted of broadening the primary and secondary templates by $v \sin i$ of $1 \mathrm{~km} \mathrm{~s}^{-1}$ and $3 \mathrm{~km} \mathrm{~s}^{-1}$, respectively, on top of their initial broadening of $3.6 \mathrm{~km} \mathrm{~s}^{-1}$ and $1.4 \mathrm{~km} \mathrm{~s}^{-1}$. With this TODCOR setup, a third correlation feature corresponding to component $\mathrm{Bb}$ was visible at some orbital phases. Although not feasible with TODCOR, extracting the exact radial velocity of component $\mathrm{Bb}$ from our spectra is possible and will be the subject of a forthcoming paper (Mazeh et al. in prep.).

\section{Results}

Our radial velocities for HD $188753 \mathrm{~A}$ and HD $188753 \mathrm{Ba}$ are displayed in Fig. 1 and are listed in Table 1 available in the electronic version. Internal uncertainties for each measurement were first estimated using a one-dimensional error analysis as explained in Zucker \& Mazeh (1994). Internal uncertainties computed in this way seem correct from a relative point of view, but are too pessimistic in absolute terms when compared to external uncertainties measured by the root-mean-squares (rms) around the orbits. This is true not only for HD $188753 \mathrm{~A}$ and $\mathrm{Ba}$, but also for the few other systems we have analyzed so far with TODCOR. We consequently used the one-dimensional uncertainties as relative weights, but then rescaled them globally, forcing the match with the rms derived from the external error analysis. The internal uncertainties finally used (and given in electronic form) are those rescaled values.

The error analysis shows that the precision on our radial velocities is degraded (factor of about 2) when the velocity difference between components $\mathrm{A}$ and $\mathrm{Ba}$ is less than $3 \mathrm{~km} \mathrm{~s}^{-1}$. Note that, although not reported, Konacki probably faced the 

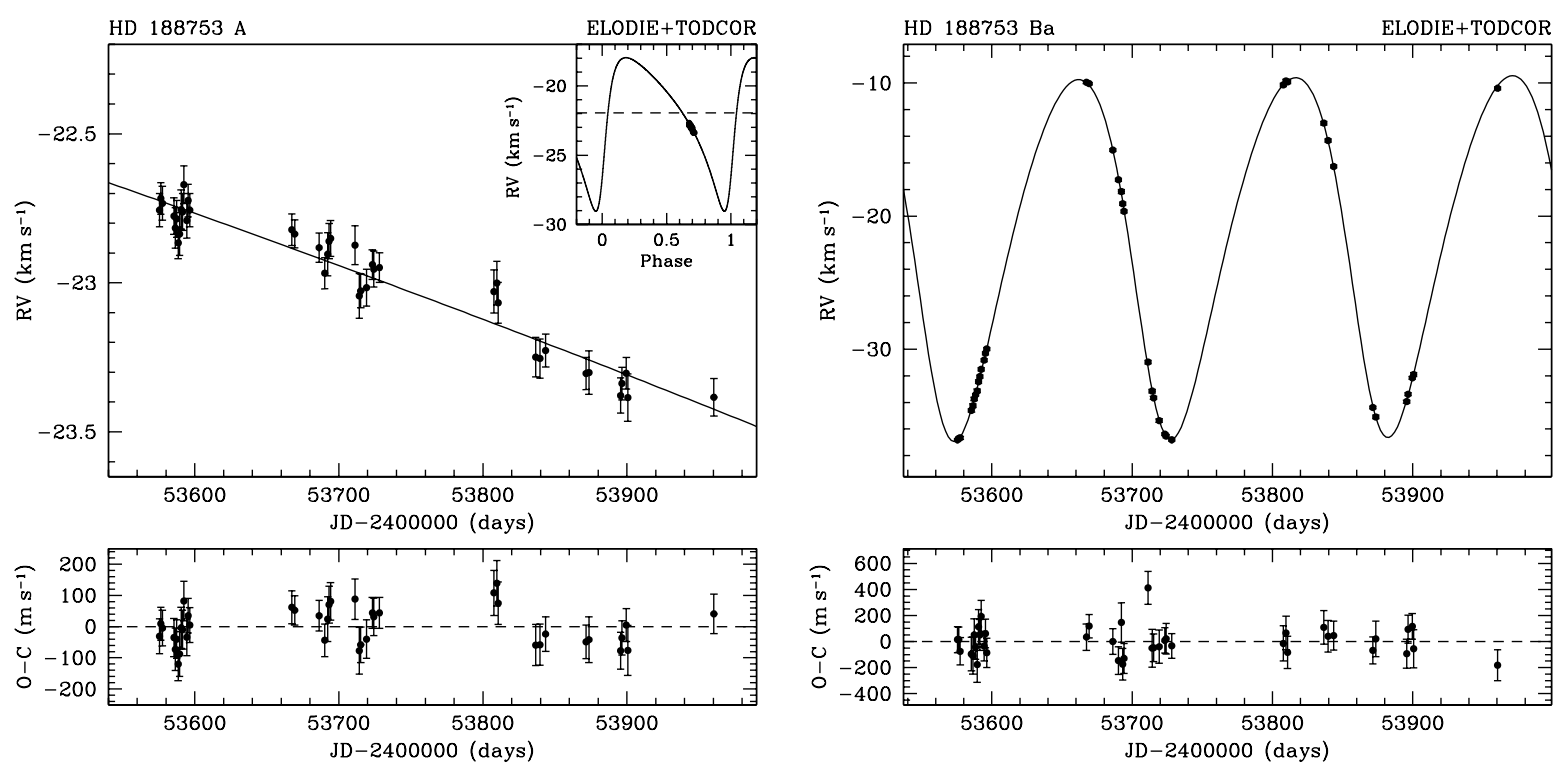

Fig. 1. Radial velocities and orbital solutions for HD 188753 A (left) and HD 188753 Ba (right). For component A, the solid line represents the 25.7-year orbital motion of the visual pair shown in full in the inset. This orbit was computed by using the elements in common with the visual orbit as fixed parameters (Söderhjelm 1999), using our radial velocities (shown as dots) to fit only the systemic velocity and the radial-velocity semiamplitude. The residuals around this long-period orbit are shown in the bottom panel. The rms is $60 \mathrm{~m} \mathrm{~s}^{-1}$. The best-fit orbital solution for component $\mathrm{Ba}$ is given in Table 2, and it includes a linear drift to take the long-period orbital motion of the AB pair into account. The bottom panel shows the residuals around this solution $\left(\mathrm{rms}\right.$ of $\left.107 \mathrm{~m} \mathrm{~s}^{-1}\right)$.

same problem, since in his measurements, taken in 7 runs spread over 466 days, the difference in radial velocity between the two brightest components is always greater than $7 \mathrm{~km} \mathrm{~s}^{-1}$, which is not likely to be a coincidence as this happens only about half of the time. In consequence, we derived orbital solutions using only our 41 measurements with a velocity difference larger than $3 \mathrm{~km} \mathrm{~s}^{-1}$.

As shown by Konacki (2005a), the spectroscopic binary with a period of 155 days detected by Griffin (1977) is the B component. Our single-lined spectroscopic orbit for HD 188753 B (including a linear drift to take the long-period $\mathrm{AB}$ motion into account) is displayed in Fig. 1. The corresponding orbital elements are listed in Table 2 . All these elements are relatively well constrained.

For the A component, the dominant motion seen in our data is a steady decrease in velocity. To check the consistency of this velocity decrease with the 25.7-year orbital motion of the $A B$ pair, we fitted our radial velocities with a Keplerian curve computed using the elements from the visual orbit (Söderhjelm 1999) as fixed parameters. The only two free parameters in the fit were thus the systemic velocity and the velocity semiamplitude, $K_{\mathrm{A}}$. The result is displayed in Fig. 1 and shows that the observed velocity decrease is indeed consistent with the orbital motion of the $\mathrm{AB}$ pair. The fit in Fig. 1 yields a velocity semiamplitude $K_{\mathrm{A}}=5.54 \pm 0.25 \mathrm{~km} \mathrm{~s}^{-1}$. Combining this value with the sum $K_{\mathrm{A}}+K_{\mathrm{B}}=9.23 \pm 0.86 \mathrm{~km} \mathrm{~s}^{-1}$ derived from the elements of the visual orbit ${ }^{1}$, we obtain a mass ratio $q_{\mathrm{AB}}=M_{\mathrm{B}} / M_{\mathrm{A}}=1.50 \pm$ 0.37 . This value is fully consistent with the masses reported by Konacki (2005a), which yield a mass ratio $q_{\mathrm{AB}}=1.54 \pm 0.12$.

In order to further study the radial-velocity variation induced by the wide orbit, we show in Fig. 2 the velocity of HD 188753 A along with the velocity of HD $188753 \mathrm{Ba}$ after having removed

${ }^{1}$ Söderhjelm (1999) does not give uncertainties on the orbital elements. Our calculation assumes uncertainties of 0.1 years on the period, of $0.02^{\prime \prime}$ on the angular semimajor axis, and of 0.03 on the eccentricity.
Table 2. Orbital parameters for the 155-day spectroscopic orbit of HD $188753 \mathrm{Ba}$, where the long-period orbital motion of the $\mathrm{AB}$ pair was taken into account by including a linear drift.

\begin{tabular}{llc}
\hline \hline Parameter & Units & Value \\
\hline$P$ & (days) & $154.56 \pm 0.08$ \\
$T$ & $(\mathrm{JD}-2400000)$ & $53405.98 \pm 0.51$ \\
$e$ & & $0.1671 \pm 0.0017$ \\
$\gamma$ & $\left(\mathrm{km} \mathrm{s}^{-1}\right)$ & $-21.625 \pm 0.034$ \\
$\omega$ & $(\mathrm{deg})$ & $136.2 \pm 1.2$ \\
$K$ & $\left(\mathrm{~km} \mathrm{~s}^{-1}\right)$ & $13.554 \pm 0.029$ \\
Linear drift & $\left(\mathrm{km} \mathrm{s}^{-1} \mathrm{yr}^{-1}\right)$ & $0.342 \pm 0.071$ \\
$a_{1} \sin i$ & $(\mathrm{AU})$ & $0.18986 \pm 0.00042$ \\
$f(m)$ & $\left(M_{\odot}\right)$ & $0.03822 \pm 0.00025$ \\
\hline$N_{\text {meas }}$ & & 41 \\
rms & $\left(\mathrm{m} \mathrm{s}^{-1}\right)$ & 107 \\
\hline
\end{tabular}

the 155-day modulation. This is presented in two panels. The left-hand panel depicts the two sets of velocities as a function of time, where the opposite slopes of the two stars can be seen easily. The right-hand panel shows a Wilson-Mazeh plot (Wilson 1941; Mazeh et al. 2002) in which the velocities of HD $188753 \mathrm{Ba}$ and HD $188753 \mathrm{~A}$ are plotted against each other (with the average velocity removed from each data set). As explained in Wilson (1941), plotting the pairs of velocities corresponding to each observation in this way should result in a straight line, whose negative slope is the inverse of the mass ratio. Figure 2 shows that our data points are indeed consistent with a straight line, except for two somewhat discordant points. Fitting the plot in Fig. 2 with a linear relation through the origin, taking individual uncertainties on both axes into account and ignoring the two discordant measurements, we obtain a mass ratio $q_{\mathrm{AB}}=1.54 \pm 0.19$. This result is in very good agreement with the two values reported previously, emphasizing the consistency 

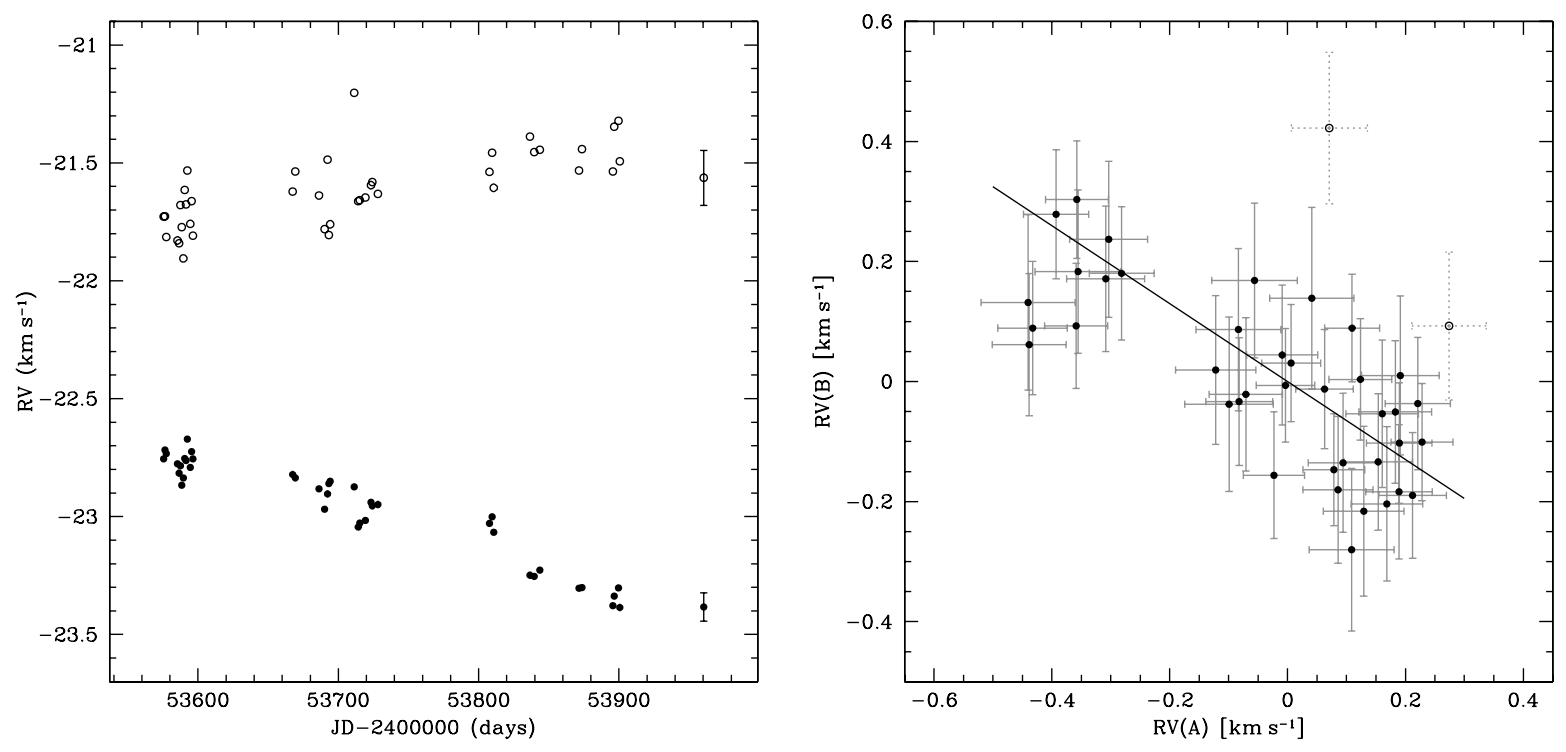

Fig. 2. Left: radial velocities for HD 188753 A (dots) and for HD 188753 Ba after having removed the 155-day modulation (open circles). For the sake of clarity, only a typical error bar is displayed on the last measurement of each component. Right: Wilson-Mazeh diagram showing the radial velocities of $\mathrm{HD} 188753 \mathrm{Ba}$ (as displayed on the left panel but with the average velocity subtracted) versus the radial velocities of HD 188753 A (again with the average velocity subtracted). As expected, our measurements are consistent with a straight line, except for two somewhat discordant points represented by open symbols and dotted error bars. The straight line shown on this plot is the result of a least-square fit taking individual uncertainties on both axes into account and ignoring the two discordant measurements. The slope of this best-fit line yields a mass ratio $q_{\mathrm{AB}}=1.54 \pm 0.19$.

between our radial velocities, the visual orbit determined by Söderhjelm (1999), and the masses derived by Konacki (2005a).

To search for evidence of the planet found by Konacki (2005a), we computed the Lomb-Scargle periodogram of the residuals around the long-period $\mathrm{AB}$ orbit for component $\mathrm{A}$. This is shown in Fig. 3. Contrary to our expectations, we did not find any significant signal in this periodogram, in particular at a frequency of 0.30 days $^{-1}$, corresponding to the 3.35-day planet reported by Konacki (2005a). That is, our velocities for HD 188753 A show no sign of a short-period signal in addition to the velocity decrease related to the $A B$ orbital motion. The rms of $60 \mathrm{~m} \mathrm{~s}^{-1}$ is basically noise and can be interpreted as the precision we achieve on the radial velocity of this star.

Could it be that we missed the planet discovered by Konacki (2005a), either because of inadequate temporal sampling or because of insufficient precision? To check the adequacy of our precision and temporal sampling to detect the potential hot Jupiter around HD 188753 A, we constructed 1000 artificial velocity sets by adding a residual value (drawn at random from our residual data set for component A) to a planetary signal computed from the orbital parameters quoted by Konacki (2005a), and sampled at our own observing epochs. Note that in the planetary signal we included the linear and quadratic trends reported by Konacki (2005a) and corresponding to the 25.7-year orbital motion. We then analyzed each of these artificial velocity sets by subtracting a linear drift representing the long-period AB orbital motion and by computing the Lomb-Scargle periodogram of the residuals. All the mock residual sets displayed an $\mathrm{rms}$ $\geq 105 \mathrm{~m} \mathrm{~s}^{-1}$, that is, about twice larger than the value actually observed. In the Lomb-Scargle periodogram, all the artificial sets displayed a marked peak at the expected frequency, with a false alarm probability $\leq 2 \%$. As we found the inserted periodicity in all our 1000 simulations, there is less than a $0.1 \%$ probability than the planet discovered by Konacki (2005a) could hide in our data set. These results confirm that we indeed have an adequate temporal sampling and sufficient precision for detecting a planetary signal similar to the one reported by Konacki (2005a). From our failure to detect such a signal, we conclude that our data show no evidence of a short-period massive planet orbiting HD $188753 \mathrm{~A}$.

\section{Discussion and conclusions}

Our observations of HD 188753 confirm that this system is triple and that HD $188753 \mathrm{~B}$ is itself a spectroscopic binary with a period of 155 days. Our orbital parameters for HD $188753 \mathrm{Ba}$ are similar to those reported by Konacki (2005a), and both analyses indicate that the B component is more massive than component A. However, we failed to detect the presumed short-period planetary companion to HD $188753 \mathrm{~A}$, despite our clear ability to do so. Instead, our data and analysis show only a steady drift in radial velocity, consistent with the 25.7-year orbital motion of the visual pair.

Although we disagree with Konacki (2005a) regarding the existence of a hot Jupiter orbiting HD 188753 A, Konacki's velocities are consistent with our analysis and conclusions, provided we admit that the precision on these velocities is not $15 \mathrm{~m} \mathrm{~s}^{-1}$, but rather about $140 \mathrm{~m} \mathrm{~s}^{-1}$. Moreover, it should be noted that Konacki's velocities do not support the planetary hypothesis any more than do our own data. Indeed, when plotting a Lomb-Scargle periodogram of the residuals of his measurements around the quadratic drift he quotes, the peak at 3.35 days has a false alarm probability of $24 \%$, and so is not significant. Even if the planetary solution reported by Konacki (2005a) satisfactorily fits his 11 velocity points ( 9 epochs), we consider that this model is very likely an artificial high-frequency fit to a small data set with large residuals. We thus conclude that there 


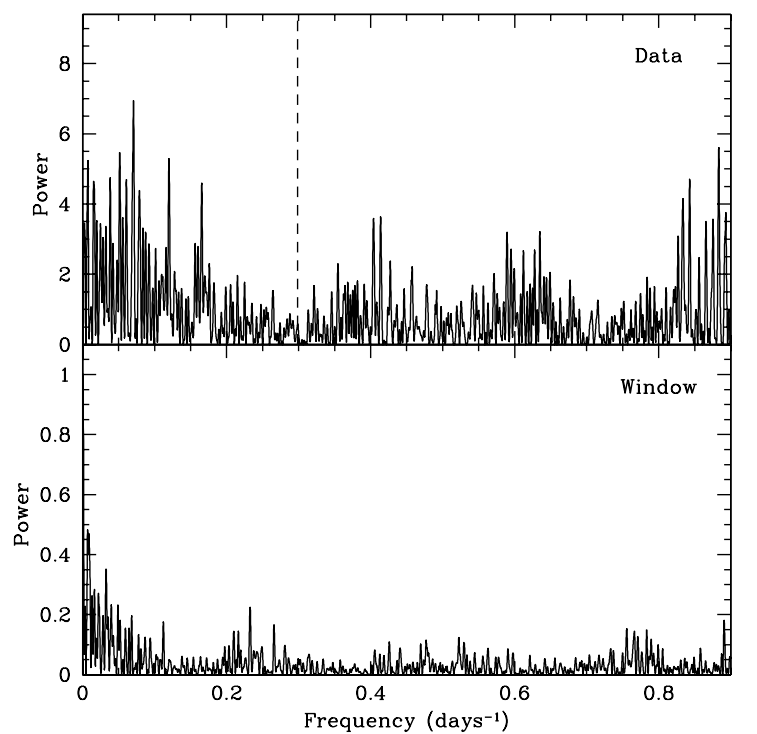

Fig. 3. Lomb-Scargle periodogram of the residuals around the 25.7-year orbital motion for HD $188753 \mathrm{~A}$. The bottom panel shows the corresponding window function. The power is a measure of the statistical significance of a periodic signal at a given frequency. For this data set, the $1 \%$ false alarm probability corresponds to a power of 9.4 and is represented by the top of the box. The highest peak at $f=0.0707$ days $^{-1}$ has a false alarm probability of $28 \%$. The dashed line denotes the frequency of the planetary signal reported by Konacki (2005a).

is currently no convincing evidence of a close-in giant planet around HD $188753 \mathrm{~A}$.

The example of HD 188753 shows that extracting precise radial velocities for the primary component of a spectroscopic binary can be challenging and that estimating the precision we really achieve on these velocities is even harder. Using our multi-order TODCOR algorithm that is specially designed to detect faint secondaries, we typically achieve a precision of $10-30 \mathrm{~m} \mathrm{~s}^{-1}$ on the radial velocity of the primary component of a double or triple system with a magnitude difference $3<\Delta V<5.5$; see Zucker et al. (2004) or Eggenberger et al. (2006) for examples. In this respect, the precision of $60 \mathrm{~m} \mathrm{~s}^{-1} \mathrm{ob}-$ tained here for HD 188753 A seems abnormally poor. One possible reason may be the lower magnitude difference $(\Delta V=0.75)$ coupled to the large-amplitude 155-day modulation induced by component $\mathrm{Ba}$. Another possibility may be the presence of the spectrum of component $\mathrm{Bb}$ in our data, which we ignored in the present analysis. To take this additional contribution into account and to properly analyze triple-lined systems, we are developing a three-dimensional correlation algorithm (Mazeh et al. in prep.). Apart from enabling us to extract all the information contained in our composite spectra, this new algorithm should prove useful in identifying the main factor that limits our current precision on the velocities of HD $188753 \mathrm{~A}$.

While three planets have been found so far in binaries with a separation of $\sim 20 \mathrm{AU}$ (Sect. 1), HD $188753 \mathrm{Ab}$ was the only planet known to reside in a tighter system. If HD $188753 \mathrm{Ab}$ is removed from the list of planetary candidates, it may be tempting to associate the value of $\sim 20 \mathrm{AU}$ with a "minimum separation" for considering that a binary (or a pair) possibly harbors a circumprimary giant planet. Nevertheless, we must bear in mind that selection effects are still strongly against planet detection in the closest binaries. Hence, the "limit" at $\sim 20$ AU may alternatively reflect our present detection capabilities, or rather their limits. In order to investigate the occurrence of planets in the closest binaries, we must therefore first characterize our detection capabilities in detail for various types of systems. This work is in progress, and definitive results from the two planet search programs targeting spectroscopic binaries (Eggenberger et al. 2003; Konacki 2005b) should provide stronger constraints on the reality of the 20-AU "limit".

Acknowledgements. We thank X. Delfosse and F. Galland for scheduling arrangements and for carrying out some of the observations of HD 188753. We warmly thank F. Bouchy, R. da Silva, B. Loeillet, C. Moutou, F. Pont, N. Santos, and D. Ségransan for carrying out observations of HD 188753 during their observing runs. We thank S. Zucker and B. Markus for their involvement and help in the development of the multi-order version of TODCOR. We thank Y. Tsodikovich for performing the least-square fit in the Wilson-Mazeh plot. We acknowledge support from the Swiss National Research Foundation (FNRS), the Geneva University, and the Israeli Science Foundation through grant no. 233/03. This work has made use of the ORBIT code developed by T. Forveille (Forveille et al. 1999).

\section{References}

Baranne, A., Queloz, D., Mayor, M., et al. 1996, A\&AS, 119, 373 Boss, A. P. 2006, ApJ, 641, 1148

Delfosse, X., Forveille, T., Perrier, C., \& Mayor, M. 1998, A\&A, 331, 581

Delfosse, X., Forveille, T., Beuzit, J.-L., et al. 1999, A\&A, 344, 897

Eggenberger, A., Udry, S., \& Mayor, M. 2003, in Scientific Frontiers in Research on Extrasolar Planets, ed. D. Deming, \& S. Seager, ASP Conf. Ser., 294, 43 Eggenberger, A., Udry, S., \& Mayor, M. 2004, A\&A, 417, 353

Eggenberger, A., Udry, S., Mayor, M., et al. 2006, in Multiple Stars Across the H-R Diagram, ESO Astrophysic Symposia, ed. S. Hubrig, M. Petr-Gotzens and A. Tokovinin, in press, available online at http: //www .eso.org/gen-fac/meetings/ms2005/eggenberger.pdf Els, S. G., Sterzik, M. F., Marchis, F., et al. 2001, A\&A, 370, L1 Forveille, T., Beuzit, J.-L., Delfosse, X., et al. 1999, A\&A, 351, 619 Griffin, R. F. 1977, The Observatory, 97, 15

Hatzes, A. P., Cochran, W. D., Endl, M., et al. 2003, ApJ, 599, 1383

Jang-Condell, H. 2007, ApJ, 654, 641

Konacki, M. 2005a, Nature, 436, 230

Konacki, M. 2005b, ApJ, 626, 431

Lagrange, A.-M., Beust, H., Udry, S., Chauvin, G., \& Mayor, M. 2006, A\&A, 459,955

Mayer, L., Wadsley, J., Quinn, T., \& Stadel, J. 2005, MNRAS, 363, 641

Mazeh, T., Prato, L., Simon, M., et al. 2002, ApJ, 564, 1007

Mugrauer, M., \& Neuhäuser, R. 2005, MNRAS, 361, L15

Mugrauer, M., Neuhäuser, R., Seifahrt, A., Mazeh, T., \& Guenther, E. 2005, A\&A, 440, 1051

Nelson, A. F. 2000, ApJ, 537, L65

Neuhäuser, R., Mugrauer, M., Fukagawa, M., Torres, G., \& Schmidt, T. 2007, A\&A, 462, 777

Perrier, C., Sivan, J.-P., Naef, D., et al. 2003, A\&A, 410, 1039

Pfahl, E. 2005, ApJ, 635, L89

Pfahl, E., \& Muterspaugh, M. 2006, ApJ, 652, 1694

Pickles, A. J. 1998, PASP, 110, 863

Portegies Zwart, S. F., \& McMillan, S. L. W. 2005, ApJ, 633, L141

Queloz, D., Mayor, M., Weber, L., et al. 2000, A\&A, 354, 99

Raghavan, D., Henry, T. J., Mason, B. D., et al. 2006, ApJ, 646, 523

Söderhjelm, S. 1999, A\&A, 341, 121

Torres, G. 2007, ApJ, 654, 1095

Wilson, O. C. 1941, ApJ, 93, 29

Zucker, S., \& Mazeh, T. 1994, ApJ, 420, 806

Zucker, S., Mazeh, T., Santos, N. C., Udry, S., \& Mayor, M. 2003, A\&A, 404, 775

Zucker, S., Mazeh, T., Santos, N. C., Udry, S., \& Mayor, M. 2004, A\&A, 426, 695 
A. Eggenberger et al.: No evidence of a hot Jupiter around HD 188753 A, Online Material p 1

\section{Online Material}


A. Eggenberger et al.: No evidence of a hot Jupiter around HD 188753 A, Online Material p 2

Table 1. Radial velocities for HD 188753 A and HD 188753 Ba.

\begin{tabular}{|c|c|c|c|c|}
\hline (JD-2 400000$)$ & $\begin{array}{c}\text { Radial velocity } \\
\text { of HD } 188753 \mathrm{~A} \\
\left(\mathrm{~km} \mathrm{~s}^{-1}\right)\end{array}$ & $\begin{array}{c}\text { Uncertainty } \\
\left(\mathrm{km} \mathrm{s}^{-1}\right)\end{array}$ & $\begin{array}{c}\text { Radial velocity } \\
\text { of } \mathrm{HD} 188753 \mathrm{Ba} \\
\left(\mathrm{km} \mathrm{s}^{-1}\right)\end{array}$ & Uncertainty \\
\hline 53575.47219 & -22.756 & 0.056 & -36.815 & 0.100 \\
\hline 53576.46953 & -22.717 & 0.053 & -36.711 & 0.098 \\
\hline 53577.47838 & -22.733 & 0.057 & -36.662 & 0.105 \\
\hline 53585.44767 & -22.776 & 0.061 & -34.600 & 0.129 \\
\hline 53586.45691 & -22.816 & 0.068 & -34.251 & 0.141 \\
\hline 53587.40502 & -22.785 & 0.062 & -33.734 & 0.123 \\
\hline 53588.43320 & -22.866 & 0.053 & -33.427 & 0.093 \\
\hline 53589.48392 & -22.836 & 0.072 & -33.138 & 0.135 \\
\hline 53590.46733 & -22.754 & 0.066 & -32.440 & 0.133 \\
\hline 53591.50460 & -22.762 & 0.062 & -32.056 & 0.119 \\
\hline 53592.42986 & -22.671 & 0.063 & -31.515 & 0.123 \\
\hline 53594.48743 & -22.791 & 0.059 & -30.826 & 0.114 \\
\hline 53595.41994 & -22.724 & 0.055 & -30.305 & 0.110 \\
\hline 53596.44843 & -22.756 & 0.056 & -29.980 & 0.112 \\
\hline 53667.37993 & -22.822 & 0.053 & -9.933 & 0.101 \\
\hline 53669.29918 & -22.836 & 0.047 & -10.044 & 0.089 \\
\hline 53686.25752 & -22.882 & 0.049 & -15.046 & 0.099 \\
\hline 53690.26372 & -22.968 & 0.052 & -17.270 & 0.106 \\
\hline 53692.26624 & -22.904 & 0.072 & -18.145 & 0.151 \\
\hline 53693.25587 & -22.860 & 0.059 & -19.072 & 0.122 \\
\hline 53694.23432 & -22.851 & 0.060 & -19.647 & 0.116 \\
\hline 53711.29621 & -22.874 & 0.065 & -30.974 & 0.126 \\
\hline 53714.24526 & -23.044 & 0.075 & -33.152 & 0.145 \\
\hline 53715.21499 & -23.027 & 0.057 & -33.653 & 0.106 \\
\hline 53719.21177 & -23.016 & 0.062 & -35.359 & 0.128 \\
\hline 53723.24701 & -22.939 & 0.050 & -36.383 & 0.098 \\
\hline 53724.23431 & -22.954 & 0.060 & -36.528 & 0.117 \\
\hline 53728.23107 & -22.949 & 0.050 & -36.814 & 0.095 \\
\hline 53807.68889 & -23.029 & 0.072 & -10.138 & 0.135 \\
\hline 53809.68173 & -23.001 & 0.073 & -9.852 & 0.129 \\
\hline 53810.67510 & -23.067 & 0.068 & -9.917 & 0.124 \\
\hline 53836.63984 & -23.249 & 0.066 & -13.014 & 0.130 \\
\hline 53839.62754 & -23.254 & 0.066 & -14.314 & 0.121 \\
\hline 53843.60439 & -23.227 & 0.055 & -16.263 & 0.111 \\
\hline 53871.58461 & -23.304 & 0.054 & -34.381 & 0.104 \\
\hline 53873.59403 & -23.301 & 0.073 & -35.092 & 0.136 \\
\hline 53895.59737 & -23.378 & 0.059 & -33.937 & 0.111 \\
\hline 53896.58051 & -23.338 & 0.055 & -33.379 & 0.108 \\
\hline 53899.58774 & -23.303 & 0.053 & -32.146 & 0.098 \\
\hline 53900.55889 & -23.385 & 0.080 & -31.905 & 0.146 \\
\hline 53960.38404 & -23.383 & 0.063 & -10.397 & 0.118 \\
\hline
\end{tabular}

